\title{
Pattern recognition of electric vehicle charging and discharging based on the closeness degree Xi Chen
}

\author{
School of North China Electric Power University, Baoding 071000, China;
}

787605053@qq.com

Keywords: closeness degree, electric vehicle, non intrusive, smart meter, pattern recognition

\begin{abstract}
In the process of the intelligent network informatization, automation, and intelligent constantly improving, the requirements of power system for the transparency of the demand side are also getting higher and higher. Real-time and accurate load equipment identification for planning, load forecasting and price adjustment of power system is very important. Electric vehicles has been rapid development in recent years. Because of the characteristics of its battery charging storage, it can be a good part of "cut peak fill in the valley" in the power system. Based on data provided by the smart meters, the electric vehicle charging and discharging characteristics has been analyzed systematically. Conventional electric vehicle charging and discharging transient and steady state parameters as the basic template is used to design load identification method of electric vehicle charging and discharging based on non intrusive.
\end{abstract}

\section{Introduction}

With the development of smart grid, electric power enterprises can get a large number of real-time data to analyze the different effects generated by the users of electric vehicles to electric power system and encourage users to charge electric vehicle in the valley, and echo electricity energy in the peak through the battery to improve the efficiency in the use of power, to improve the load curve and to achieve the goal of "cut peak and fill valley". Therefore, the research and application of the identification technology of the electric vehicle charging and discharging of the user side provide the technical support for the "cut peak and fill valley".

Smart meters can get more accurate parameters of the power grid. The non - invasive method is carried out in the pattern recognition of electric vehicles, namely at the user interface active power, reactive power, current and voltage and other parameters [1]. This non intrusive monitoring method does not interrupt the power supply of equipment, which is easy to be accepted by users, and saves the cost of installation and maintenance.

At present, the convenient communication mode and powerful data acquisition function of smart meter provide a powerful guarantee for the load monitoring and identification. Therefore, the steady step change of active power can be captured in order to identify the switching state of the load and electricity consumption of load can be calculated approximately by recording the active power and reactive power of the electric vehicle in the transient and steady state. In addition, in order to identify the load equipment more accurately, this paper uses the transient and steady state power of the electric vehicle as the characteristic to obtain the power consumption and working status of the electric vehicle.

In summary, this paper focuses on the analysis of the characteristics of electric vehicle charging and discharging behavior combined with the closeness degree method, a load identification method has been built.

\section{Basic principle}

Closeness is a measure to describe the similarity between data objects. The Euclidean distance method is simple and convenient. The Euclidean distance method is adopted in this paper.

Euclidean distance of Load waveform is: 


$$
D(x, y)=\sqrt{\frac{1}{n} \sum_{i=1}^{n}\left(x_{i}-y_{i}\right)^{2}}
$$

Normalizing the data:

$$
x_{i}^{\prime}=\frac{x_{i}}{a}, y_{i}^{\prime}=\frac{y_{i}}{b}
$$

Where $a=\max x, \quad b=\max y, D(x, y) \in[0,1]$ 。

So get closeness degree:

$$
N(x, y)=1-D(x, y)=1-\sqrt{\frac{1}{n} \sum_{i=1}^{n}\left(x_{i}^{\prime}-y_{i}^{\prime}\right)^{2}}
$$

In order to make the closeness degree between the amplitude equal the range of the above type, the closeness degree between the amplitude is defined as:

$$
N_{a}^{b}=e^{-(a / b-1)^{2}}
$$

\section{Maximum membership degree principle}

The maximum membership degree principle is defined as follows [2]:

Let $m$ fuzzy subsets $A_{1}, A_{2}, \cdots, A_{m}$ in domain $U$ Constitute a standard template library. If any of the elements $u_{0} \in U$ meets $\mu_{A_{i}}=\max \left(\mu_{A_{1}}\left(u_{0}\right), \mu_{A_{2}}\left(u_{0}\right), \cdots, \mu_{A_{m}}\left(u_{0}\right)\right), \quad u_{0}$ is considered subordinate $A_{i}$ relatively

The closeness degree is used as the membership degree function, and the expression of the comprehensive membership degree vector is obtained:

$$
L=\left[\mu_{A_{1}}\left(u_{0}\right), \mu_{A_{2}}\left(u_{0}\right), \cdots, \mu_{A_{m}}\left(u_{0}\right)\right]=W \times R
$$

$W$ as weight, $R$ as membership degree matrix, $R=\left[N_{1}, N_{2}, \cdots N_{n}\right]$ 。

\section{Identification of electric vehicles}

We used the method of combining qualitative and quantitative methods to make a preliminary attempt on the process of electric vehicle access to the power grid.

When electric vehicle is charging, it is resistance capacity of electrical appliances, while ordinary household equipments are the resistance of electrical appliances (such as electric furnace), or inductive electrical appliances (such as the refrigerator). So we can identify the charging electric vehicles through the reactive power curve.

In this paper, we take the following routine parameters as the template calculating closeness degree database of the electric vehicle: AC supply voltage is $380 \mathrm{~V}$; on-board battery's capacity is 4 $\mathrm{Ah}$. In order to reduce the time of measurement, this specification parameters of battery are taken as calculate basis [3].

\section{Electric vehicle charging process analysis}

When electric vehicles are discharging, the nominal battery voltage is $240 \mathrm{~V}$, float voltage is 270 $\mathrm{V}$, termination of the discharge voltage is $220 \mathrm{~V}$, rated capacity is $90 \mathrm{Ah}$. When charging, initial state of charge (SOC) is $40 \%$, discharging is $80 \%$. The change of the charging current is shown in Figure 1 [4].

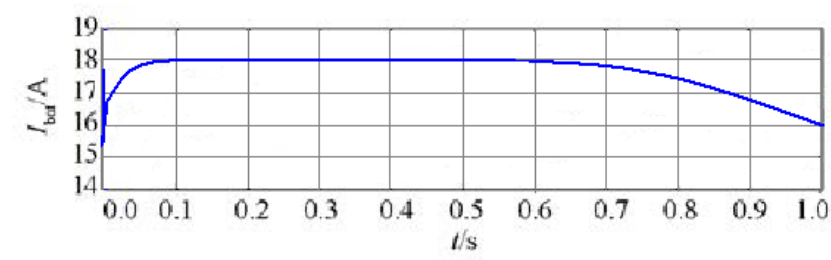

Fig. 1 change curve of charging current of electric vehicle 


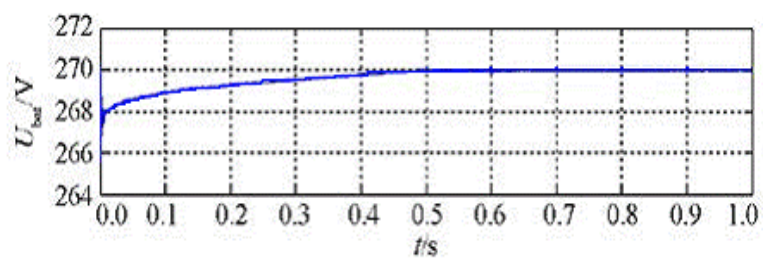

Fig. 2 voltage change curve of electric vehicle charging battery

The charging control strategy adopted achieves the purpose of constant current and constant voltage charging. After transient oscillation, the current can reach a constant current, and the battery can enter the constant current charging area [5]. In this case, we record the output power when electric vehicle is charging, and draw the following chart.

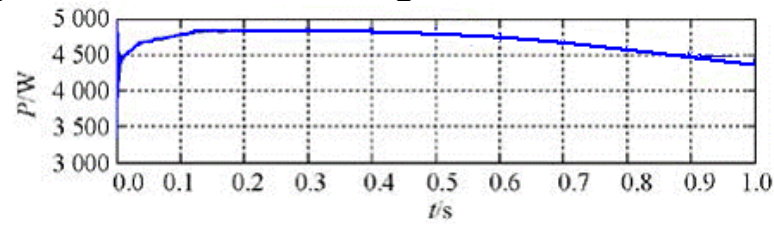

Fig.3 Output power curve of charging electric vehicle

\section{Electric vehicle discharging process analysis}

The discharge current waveform is shown in the figure below:

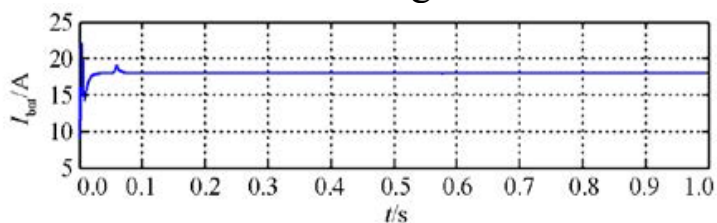

Fig. 4 curve of discharge current in electric vehicle

The output power curve of the discharge is shown in the following figure:

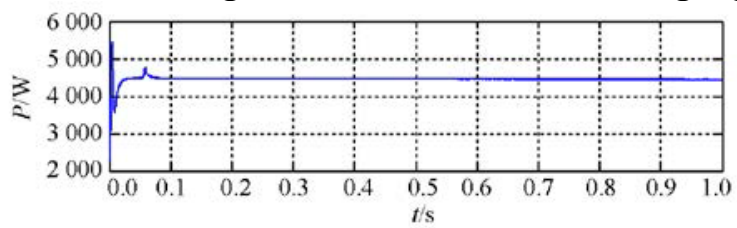

Fig. 5 output power curve of electric vehicle

Then discharge control strategy still achieves the purpose of constant current discharge. after discharge current a short-term shocks, it reaches a predetermined current value and maintain a constant to discharge. With the battery discharging, the terminal voltage decreased slowly. According to the obtained curve, the calculation of the closeness degree and the maximum membership degree can identify the electric vehicle and the status of charging and discharging.

\section{Conclusion}

Non intrusive load identification method directly obtain the required power parameters in the total interface. Without affecting the accuracy of the data acquisition, greatly reducing the workload of data acquisition, therefore it is easy to implement. At the same time, it can identify the charging and discharging status of the electric vehicle, which is of practical significance.

\section{Reference}

[1] In Hyeob Yu, won Yang, Jong Min Ko, etal. Development of Load Analysis System using Customer Load Profile Data [C]. SICE-ICASE International Joint Conference, 2006, Oct. 18-21:1557-1561

[2] Wang Qingyu, Zhang Qingqing, Zhang Gaofeng, et al. Application of spectral clustering algorithm in household load identification[J]. Electrical Measurement \& Instrumentation, 2015,52(1):119-123(in Chinese). 
[3] Liu Fagui, Zhao Juan. Fuzzy closeness degree and its application[J]. Journal of North China Institute of water conservancy and hydroelectric power, 2006,03:104-106(in Chinese).

[4] Liu Yuexian, Wang Tianyu, Yang Yayu, Wang Xiaoru. Modeling and Simulation of electric vehicle charging and discharging system[J]. Power System Protection and Control, 2014,13:70-76(in Chinese).

[5] Wu Kuihua, Sun Wei. Zhang Xiaonei, et al. Modeling of electric vehicle charging load and its effect on power system load characteristics[J]. Shandong Electric Power, 2013,05:5-8+21(in Chinese). 